\title{
THE PATHOLOGY OF RECTAL TUMOURS
}

\author{
By Basil C. Morson, M.A., D.M. \\ Consultant Pathologist and Director of the Research Department, St. Mark's Hospital, London
}

\section{Epithelial Tumours \\ Adenoma}

This is the commonest variety of rectal tumour. According to Helwig (I947) the incidence of adenomas increases with age. Approximately one person in five reaching the age of sixty has one or more tumours of the large intestine, most of these being found in the sigmoid colon and rectum. They are commoner in males than females in a proportion of about $3: 2$. The age incidence is approximately the same as for carcinoma, most of the tumours being detected in the sixth and seventh decades. They are frequently multiple.

Adenomas vary in size from sessile, dome-shaped nodules a few millimetres in diameter up to nearly round, pedunculated tumours about $I$ in. in diameter. They are rarely larger. Initially, all adenomas are sessile, but they become more pedunculated as they grow and as peristalsis attempts to propel them onwards with the intestinal contents. They also tend to become dark red as they enlarge, which is partly due to haemorrhage within the tumour and partly to secondary infection with associated congestion of small blood vessels. The surface of a typical adenoma is lobulated with intercommunicating fissures or crevices between the lobules (Fig. I).

In its histological structure the adenoma shows tubular overgrowth of the rectal epithelium. The epithelial cells vary from those seen in normal mucosa by a tendency to loss of mucus-secretion. They also have large vesicular nuclei and an increased number of mitoses. All stages of dedifferentiation may be seen from an obviously benign appearance to active proliferation and structural irregularity amounting to pre-invasive carcinoma. Once invasion is seen within a polyp it is no longer an adenoma, but a carcinoma; but some reference to its origin from a previously benign tumour can be usefully made.

Although it is now widely accepted that the rectal adenoma is a pre-cancerous lesion, it is useful to recall the evidence. In the first place, adenomas and carcinomas are found in the rectum with a similar frequency and have much the same

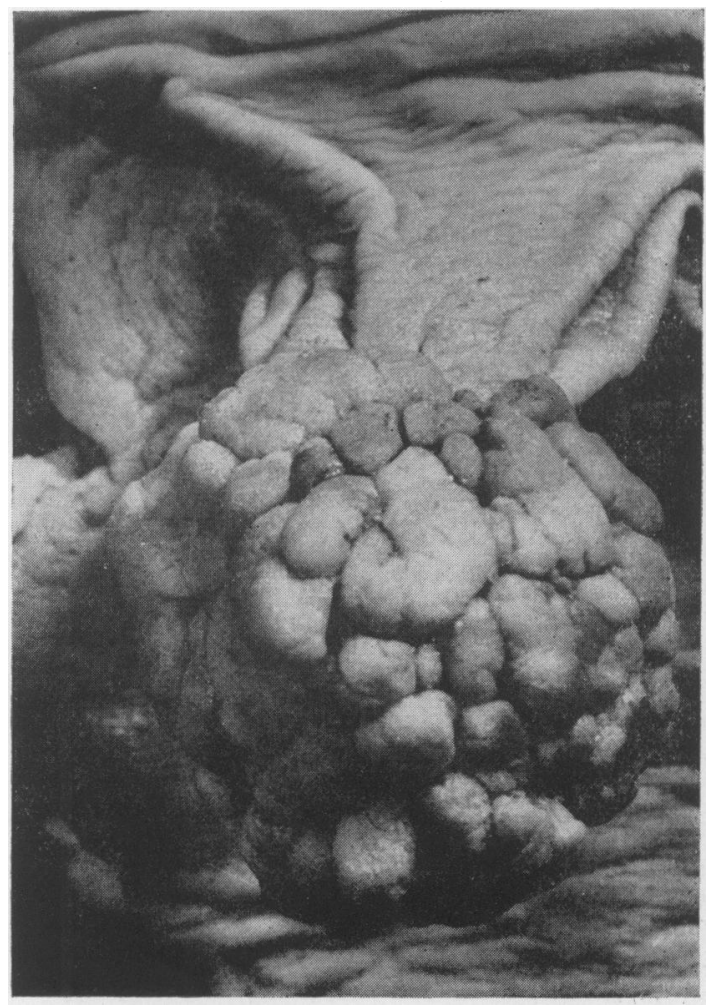

Fig. 1.-Pedunculated adenoma of rectum about $\mathbf{I}$ in. diameter.

age and sex incidence. Moreover, in operation specimens of rectal cancer one or more simple adenomata are commonly found in the neighbourhood of the primary tumour. Dukes (1926) described cases of rectal carcinoma in which there was histological evidence of an origin from a previously benign adenoma. Also, it is now a common experience for a clinically benign adenoma when examined histologically to show evidence of malignant change. Finally, it is established that patients with familial polyposis (adenomatosis) almost invariably develop carcinoma if left untreated. 


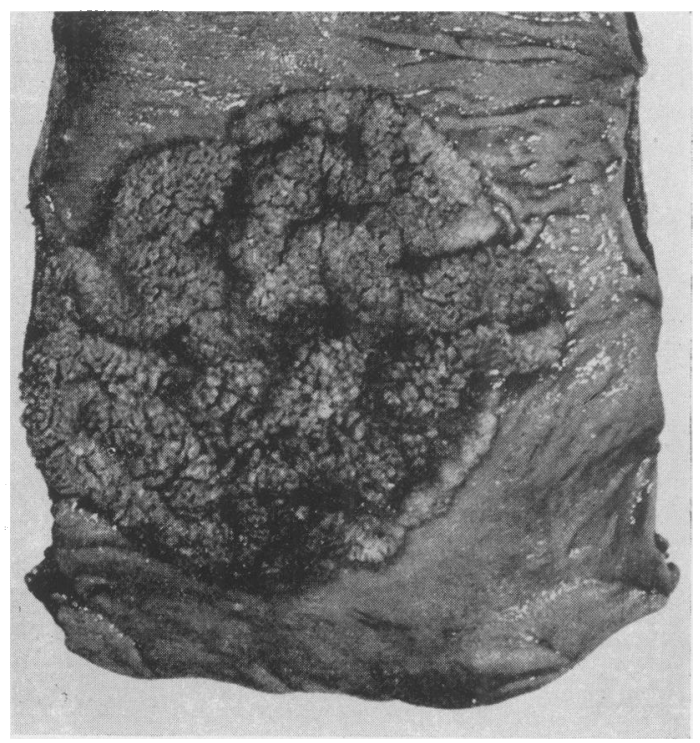

Fig. 2.-Villous papilloma of rectum about $2 \frac{1}{2}$ in. diameter.

\section{Villous Papilloma}

This tumour is much less common than the adenoma. Swinton and Doane (1952) in a review of 300 patients with benign epithelial polyps of the large intestine found that 20 (6.6 per cent.) were villous papillomas, most of them being found in the rectum. They are about equally distributed between the sexes, most of the tumours being detected in the sixth and seventh decades.

The differences between a typical adenoma and a typical villous papilloma are striking both in their pathology and behaviour. However, there are tumours which contain features of both types. This mixed group is not a very large one, but it accounts for difficulties in the differential diagnosis between an adenoma and a villous papilloma.

The villous papilloma (Fig. 2) is usually larger than the adenoma when first seen. Often it is very large, reaching 4 or 5 in. in diameter. It is always sessile, although the centre of the tumour is raised above the surface of the surrounding normal mucosa to a greater extent than at its periphery. The surface of the tumour has a convoluted appearance and is covered by delicate villous processes which make it soft and velvety to the touch. The villous papilloma is usually solitary. Microscopically it is composed of tall mucus-secreting columnar cells covering delicate, elongated villi. The amount of mucus secretion, particularly from a large tumour, may be enormous. Mucus is rich in potassium and cases have been reported of villous papillomas associated with severe potassium deficiency (Starr et al., 1956).
The fact that villous papillomas may be very large, yet benign, does not mean that they are any less prone to malignant change than the simple $\stackrel{2}{c}$ adenoma. Their size is only a reflection of their $\Rightarrow$ mode of origin. Whereas the adenoma arises from a relatively small circumscribed area of mucosa, the 0 villous papilloma usually has a wide field of origin. $\frac{\bar{D}}{\underline{D}}$ This makes the detection of early malignant change $\frac{\bar{\rho}}{\frac{\rho}{\sigma}}$ a difficult problem for both the clinician and the $\stackrel{\mathbb{Q}}{\Omega}$ pathologist. The softness and mobility of a villous 0

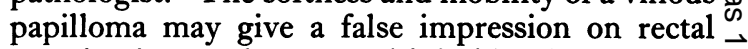
examination, and even multiple biopsies may not $\vec{\circ}$ be representative of the whole tumour. Some- $\overrightarrow{\vec{\omega}}$ times, complete local excision is necessary before $\stackrel{\omega}{\circ}$ malignant change can be excluded.

Reference has been made above to the excessive 3 . secretion of mucus by villous papillomas. This characteristic is passed on when they become malignant. In the St. Mark's series of villous 8 papillomas showing malignant change, about a $\overrightarrow{0}$ third of the carcinomas are of the mucus-secreting or colloid variety. This is three times the in- $\frac{}{3}$ cidence of mucus-secreting carcinomas in the general series of St. Mark's cases.

\section{Familial Polyposis}

This is an hereditary disease in which the colos and rectum are covered by large numbers 0 adenomata of different sizes and at different stage. of development. Polyposis is transmitted by dominant gene, the affected parent passing it on to approximately half of his or her children. At St. Mark's Hospital research into familial polyposis has been carried on for the past 30 years. The $\stackrel{\mathbb{\Omega}}{2}$ pathology of the disease in 156 patients from $41 \overrightarrow{\overrightarrow{0}}$ families has been described by Dukes (1952). The 3 adenomata of familial polyposis develop in postnatal life, the average age of onset of symptoms being about 20 years, but it is rare for the first $\bar{\partial}$ symptoms to occur before the age of ten or after 3 . the age of forty. Carcinoma of the colon or rectum is almost certain to develop in a patient with familial polyposis, the average age of onset being about 35 years compared with an average age of 60 years for patients with non-familial carcinoma.

\section{Congenital Polyps}

There are two rare varieties of rectal polyp with N which the simple adenoma may be confused. N Firstly, there is the congenital mucous polyp. This N is not a neoplasm but a hamartoma, and is there- $\omega$ fore not a pre-cancerous lesion. It is composed of rectal tubules lined by well-differentiated mucus- $\stackrel{\varrho}{\subset}$ secreting epithelium lying in a stroma of connec- $\stackrel{\infty}{\infty}$ tive tissue which resembles primitive mesenchyme in its histological structure. The tubules tend to become dilated with excess mucus secretion, and 
the cut surface of the polyp may be obviously cystic. Furthermore, the amount of stroma is much greater than in a simple adenoma. This congenital polyp may be multiple, and is a rare cause of bleeding in children and young adults.

A second rare type of rectal polyp is found in the Peutz-Jeghers syndrome. This syndrome consists of muco-cutaneous pigmentation associated. with gastro-intestinal polyposis (Dormandy, 1958), although a minority of patients have rectal tumours. In their histological structure the Peutz-Jeghers polyps show normal intestinal epithelium covering branching bands of smooth muscle. The relationship of the epithelium to the smooth muscle is the same as that between the epithelium and the muscularis mucosa in normal mucous membrane. There is no abnormal epithelial proliferation and the appearances are those of a hamartoma and not a true neoplasm. It follows that the PeutzJeghers polyp is not a pre-cancerous lesion.

\section{Carcinoma}

Rectal carcinoma is one of the commoner forms of malignant disease. There are about 5,500 deaths per annum in England and Wales, which is 6 per cent. of all forms of cancer, the average age at death being 69 years (Registrar General, 1956). At St. Mark's Hospital the average age of patients with rectal cancer at the time of diagnosis is about 60 years and the sex incidence about $2: I$ in favour of males.

Age is important in the study of the natural history of rectal cancer. Thus, the incidence of lymphatic metastases in patients under 40 years of age is over 70 per cent., and falls to only about 40 per cent. in the eighth decade. Furthermore, the study of the relationship between age and histological grade shows that the average age of patients with low-grade carcinoma is greater than the average age of those with high-grade carcinoma.

Rectal carcinoma presents either as an ulcerating or as a protuberant tumour. It occurs with equal frequency in the upper, middle and lower thirds of the rectum. Furthermore, there is no difference in the pathological features, such as grade of malignancy and extent of spread, at the three sites. It is not surprising, therefore, that the prognosis at the different levels of the rectum after radical surgical treatment is very similar.

About 5 per cent. of patients with rectal cancer develop more than one growth of the large intestine. However, the incidence of multiple malignancy is much higher in patients with carcinoma following familial polyposis and ulcerative colitis.

About 87 per cent. of rectal carcinomas are adenocarcinomas of varying degrees of differentiation. A further ro per cent. approximately are mucus-secreting adenocarcinomas, so-called be- cause they contain substantial quantities of mucus. The remaining 3 per cent. includes purely anaplastic tumours (carcinoma simplex) and signet-ring cell carcinoma which is really anaplastic mucussecreting carcinoma. For many years it has been customary at St. Mark's Hospital to divide rectal carcinoma into three histological grades of malignancy-low, average and high. About 20 per cent. are a low grade of malignancy, 50 per cent. average and 30 per cent. high grade. That this is a valid means of assessing the approximate rate of growth of rectal carcinoma is shown by comparing the grade of malignancy with the extent of spread in operation specimens. Whereas the incidence of lymphatic metastases is only 25 per cent. in lowgrade carcinoma, it is about 50 per cent. in average grade, and nearly 80 per cent. in high-grade carcinoma. Moreover, Dukes and Bussey (1958) have shown that the histological grade of malignancy influences the survival rate of patients with rectal carcinoma. The corrected five-year survival rate for patients with low-grade tumours is nearly 80 per cent., for those of average grade about 60 per cent. and for high-grade malignancy only 29 per cent. The five-year survival rate for purely anaplastic carcinoma and for signet-ring cell tumours is worse still.

The A, B, C, or Dukes Classification of rectal carcinoma is based on the extent of spread in operation specimens. It has proved to be a con-ca venient method of relating pathology and prognosis. Dukes and Bussey (1958) have recently given an account of the extent of spread of rectal cancer and its effect on prognosis in a large series of cases. The corrected five-year survival rate of A cases (growth limited to the rectum with no extra-rectal spread or lymphatic metastases) is 97.7 per cent. The five-year survival of $B$ cases (spread by direct continuity into extra-rectal tissues without lymphatic metastases is 77.6 per cent., and for $\mathrm{C}$ cases (lymphatic metastases present) is 32 per cent. The importance of observations on lymphatic spread can be seen by comparing the survival of $B$ and $C$ cases.

Most patients with rectal cancer die from secondary deposits, usually in the liver, but also in the lungs, brain and bones. It is justifiable to presume that these secondary deposits are usually the result of metastases via the veins draining the region of the primary growth. In operation specimens it is sometimes possible to detect venous spread in the form of a solid cord of growth growing along the lumen of a vein in direct continuity with the primary tumour. This is known as visible venous spread, and must be distinguished from microscopic venous spread which cannot be detected by careful dissection. Visible venous spread has been detected in 11 per cent. of 1,795 
consecutive operation specimens at St. Mark's Hospital. The discovery of this form of spread of rectal cancer in an operation specimen worsens the prognosis only slightly (Dukes and Bussey, 1958).

Venous spread in rectal cancer may also be detected in histological sections of the primary tumour and by the cytological demonstration of tumour cells in blood taken from the superior haemorrhoidal vein. By these methods it is possible to detect venous spread in a high proportion of cases.

An account has already been given of the precancerous nature of benign epithelial tumours of the rectum and familial polyposis. Another disease which is prone to the development of carcinoma is chronic ulcerative colitis. The incidence of cancer following colitis varies with the severity and duration of the disease. Weckesser and Chinn (1953) found the average duration of symptoms of ulcerative colitis prior to the development of cancer to be about ro years. At St. Mark's Hospital the incidence of cancer of the colon or rectum in operatior specimens is about 5 per cent.

The pathology of carcinoma arising in a patient with colitis is characteristic. Firstly, the tumours are often multiple. Secondly, they are flat and infiltrating, with an ill-defined edge resembling linitis plastica of the stomach. Lastly, most of them are mucus-secreting carcinomas of a highgrade malignancy, often showing signet-ring cell formation. Generally the prognosis is poor.

\section{Carcinoid}

This is an uncommon type of rectal tumour. It presents as a small, hard submucosal nodule about I cm. diameter and is usually found accidentally. It may be mistaken for an adenoma or a carcinoma. The diagnosis is made by biopsy which shows a tumour composed of small cells containing nuclei which are very regular in size and shape and contain few mitoses. The cells are arranged in solid clumps which resemble those seen in the ileocaecal type of carcinoid, but there are also areas with an adenomatous pattern, and others show ribbon-like festoons of columnar cells as described by Stout (1942). Infiltration of the surrounding tissues is always present, but the cytoplasmic granules characteristic of the ileo-caecal type of carcinoid are seldom seen (Morson, 1958).

This small submucosal variety of rectal carcinoid is a very slowly growing malignant tumour, which is most unlikely to develop metastases. Experience at St. Mark's Hospital suggests that local excision is adequate treatment. However, if a carcinoid is greater than $I \mathbf{~ c m}$. diameter and shows ulceration, metastases may have occurred and a radical operation is necessary. Such a case has been reported by Gabriel and Morson (1956).
Histologically, this showed a degree of anaplasia not seen in the small submucosal variety of carcinoid. The patient had liver deposits at the time of operation and died three years later.

No case has yet been reported of a carcinoid of the rectum with the clinical syndrome of cutaneous flushing, diarrhoea and pulmonary stenosis due to excessive secretion of 5-hydroxytryptamine (5-H.T.). Moreover, urine tests performed on cases of rectal carcinoid at St. Mark's Hospital have never shown any excess of urinary 5 -hydroxyindole acetic acid, the excretion product of $5-\mathrm{H}$.T. This is probably explained by the lack of cytoplasmic granularity in rectal carcinoids, for it is believed that these granules are the morphological basis of $5-\mathrm{H}$.T. production.

\section{Tumours of Lymphoid Tissue Lymphoma}

In the rectum the term lymphoma is used to describe a relatively small submucous tumour which is quite benign in its behaviour. It appears to have no relationship to lymphosarcoma. It is an uncommon tumour, and only 87 cases have been seen at St. Mark's Hospital in the last 28 years. It is commoner in men than women and the average age of patients at the time of diagnosis is 36 years. Over 60 per cent. of all lymphomaso are found in the third and fourth decades of life.

Rectal lymphoma presents as a round, smooth nodule, which is entirely submucous in position. It is firm in consistency, and is rarely more than $I$ in. diameter. Most of the tumours are found in the lower third of the rectum and just above the muco-cutaneous junction of the anal canal. They may be multiple. An analysis of the St. Mark's cases shows that 80 per cent. of the tumours were found incidentally during rectal examination for other conditions, mainly of an inflammatory nature. This observation, taken in conjunction with the low average age of patients with rectal lymphoma, suggests that it is an inflammatory rather than a neoplastic condition. Furthermore, a ' follow-up ' of the St. Mark's cases has revealed no evidence of recurrence and no subsequent history of lymphosarcoma.

Lymphoma arises as a result of hyperplasia of the rectal lymphoid follicles. The tumours are composed of well-differentiated lymphoid tissue showing follicle formation and covered by attenuated mucous membrane. The appearance resembles a lymph node, but without any lymph sinuses or capsule. There is no evidence of active inflammation, no increase in the number of mitotic figures or other evidence of neoplasia.

\section{Lymphosarcoma}

This is a very rare form of rectal malignancy 
which presents either as a solitary rectal ulcer or as generalized disease of the lymphoid follicles of the rectum and colon.

The lymphosarcomatous ulcer of the rectum does not differ from carcinoma in its gross appearance, and the diagnosis of a tumour of lymphoid tissue is made by biopsy. The histological appearances have to be distinguished from benign lymphoma in particular and this may be extremely difficult, especially if the lymphosarcoma is welldifferentiated. However, the presence of a tumour greater than $\mathrm{I}$ in. diameter which is also ulcerating through the rectal mucosa supports the diagnosis of malignancy. Further, a search should be made for enlarged lymph glands elsewhere in the body and one removed for confirmation of the diagnosis.

Generalized lymphosarcomatous disease of the lymphoid follicles of the rectum and colon presents as a diffuse intestinal polyposis which must be distinguished clinically from familial adenomatosis. The polyps in this diffuse form of lymphosarcoma are composed of proliferating lymphoid tissue covered by attenuated mucosa. They are rounded tumours which become pedunculated as they grow larger. There may be an associated lymphatic leukaemia.

\section{Reticulum Cell Sarcoma}

Only three cases have been studied at St. Mark's Hospital in the last 30 years. They were very large tumours involving most of the rectum and spreading widely into the peri-rectal tissues and lymph glands. The prognosis is very poor.

\section{Tumours of Smooth Muscle Leiomyoma}

Only three examples of this benign tumour have been recorded at St. Mark's Hospital. They were small, hard submucous nodules. Microscopically they contained well-differentiated smooth muscle fibres and appeared to be arising from the muscularis mucosae. Leiomyoma arising within the muscularis externa has also been described.

\section{Leiomyosarcoma}

This is another rare form of rectal malignancy which arises from the smooth muscle in the rectal wall. Typically, it presents as a nodular, protuberant swelling which is partly covered by intact mucosa, but shows central ulceration. It can be distinguished from carcinoma because it obviously arises from the deep tissues of the rectal wall. Microscopically, the tumours are composed of interlacing bands of smooth muscle fibres which are mostly well-differentiated and of a low grade of malignancy. They spread principally by direct extension into the peri-rectal fat and by the veins, giving rise to metastases in the liver and lungs.
Lymphatic spread is only found in the very anaplastic forms of leiomyosarcoma. A study of the St. Mark's cases suggests that the ultimate prognosis is poor, the patients dying from local recurrence and secondary deposits in the liver and lungs. However, most cases of rectal leiomyosarcoma are of a relatively low grade of malignancy and more than half the patients have survived five years.

\section{Miscellaneous Rare Tumours Haemangioma}

Cavernous haemangioma may be either circumscribed or diffuse (Gabriel, I945). It involves principally the sub-mucosa of the rectum, appearing as a collection of dark red lobular swellings covered by attenuated mucous membrane. The lesion may involve the whole of the rectum, extending into the anal canal and up the sigmoid colon. In these diffuse cases the blood vessels in the perirectal fat and sigmoid mesentery may also show angiomatous changes. The condition is really a congenital abnormality and there is no neoplastic proliferation. However, because of secondary haemorrhage and technical difficulties in the surgical treatment, the prognosis of the diffuse cases of rectal angioma is poor.

\section{Lipoma}

Submucous lipoma of the rectum has been described, but is extremely rare. It presents as a? pedunculated tumour, prolapsing out of the anus. It is covered by intact mucosa and the cut surface shows yellow fatty tissue.

\section{Endometrioma}

This tumour is, of course, not a neoplasm, but its inclusion in any classification of rectal tumours is justified because it presents as a hard mass in the rectum and may be confused with carcinoma. The histological appearances in the rectal biopsy of an endometrioma may also be difficult to interpret. The tumour is submucous in position and covered by intact mucous membrane. Its cut surface shows localized smooth muscle hypertrophy around small, blood-filled cysts.

\section{Secondary Tumours of the Rectum}

Any growth which spreads into the pouch of Douglas or recto-vesical pouch may ulcerate into the rectum. This is not uncommon in carcinoma of the pelvic colon which may prolapse into the recto-vesical pouch with secondary ulceration through the rectal wall.' Secondary involvement of the rectum may also occur in this way with carcinoma of the bladder and ovary. Cervical cancer may spread through the rectal wall, but this happens only at an advanced stage of the disease. 
Despite the close anatomical relationship between the rectum and the prostate it is uncommon for prostatic cancer to invade the rectal wall, except in advanced disease. It is probable that the fascia of Denonvilliers provides an effective barrier to the backward spread of prostatic cancer. On rectal examination it presents either as an anterior submucous mass, a tubular stricture or, rarely, as a rectal ulcer. The diagnosis may be suspected clinically, and is confirmed by rectal biopsy and possibly a raised level of serum acid phosphatase. In view of the differences in treatment it is obviously important to distinguish between primary rectal cancer and secondary invasion of the rectal wall by prostatic carcinoma.

Squamous cell carcinoma, arising from the upper part of the anal canal, may spread upwards into the rectum and present as a primary rectal tumour. The examination of biopsy material will distinguish it from adenocarcinoma. Another type of anal malignancy which shows preferential local spread upwards into the rectum is the rare malignant melanoma which always arises from the upper part of the squamous-lined portion of the anal canal. It usually presents more as a rectal than an anal tumour, but its origin from the squamous epithelium of the anal canal cannot be disputed, and it is, therefore, classified with tumours of the anus.

\section{BIBLIOGRAPHY}

DUKES, C. E. (1926), Brit. F. Surg., 13, 720.

DUKES, C. E. (1952), Ann. Eugen. (Camb.), 17, 1. DUKES, C. E., and BUSSEY, H. J. R. (1958), Brit. F. Cancer. In
Press.

DORMANDY, T. L. (1958), 'Modern Trends in Gastroenterology,' Second Series. 'Edited by F. Avery Jones.

GABRIEL, W. B. (1945), 'The Principles and Practice of Rectal Surgery,' Third Edition.

GABRIEL, W. B., and MORSON, B. C. (1956), Proc. roy. Soc. Med., 49, 472.

HELWIG, E. B. (1947), Surg. Gynec. Obstet. 84, 36.

MORSON, B. C. (1958), 'Modern Trends in Gastro-enterology,' Second Series. Edited by F. Avery Jones.

REGISTRAR-GENERAL (1956), ' Statistical Review of England and Wales.

STARR, A., MUELLER, S., and McKITTRICK, J. R. (1956), Arch. Surg. (Chicago), 73, 995.

STOUT, A. P. (1942), Amer. F. Path., 18, 993.

SWINTON, N. W., and DOANE, W. A. (1952), Surg. Clin. N. Amer., 32, 923.

WECKESSER, E. C., and CHIN, A. B. (1953), f. Amer. med. Ass." I52, 905 .

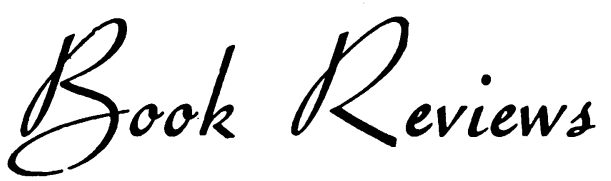

\section{CLINICAL BIOCHEMICAL METHODS}

By A. L. Tarnoky. Pp. 239, figs. i I. London: Hilger \& Watts Ltd. 50 s.

This well-produced volume is a bench book intended primarily for the biochemical technician. Instructions are clearly given, with a minimum of explanation and no interpretation. There are three pages of references and both an author and a subject index. The methods are those used in the author's laboratory in the Royal Berkshire Hospital; this means that they are well tried and that the author can vouch for their reliability, but it somewhat restricts his range. None is included for the estimation of iron, for instance. The use of the manometric Van Slyke apparatus is described for estimation of the alkali reserve (sic); it is surprising to find this included with a method for plasma $\mathrm{pH}$ which employs the Lovibond comparator. The book also presupposes that a Hilger absorptiometer will be used; surely it would be more in keeping with such an instrument to recommend the construction of standard curves? This omission is surprising in view of the inclusion of a short but useful chapter on analytical error.

A chapter on the choice and care of laboratory glassware would be useful. The book is very clearly printed and set out, with a large page and ample margins; it might have been helpful to have blank pages at intervals for notes.

\section{EMERGENCY SURGERY}

By Hamilton Bailey, F.R.C.S., F.R.S.E. Seventh Edition. Pp. I21 3, with 1576 illustrations. Bristol: John Wright and Sons Ltd. 1958. £9 9s. od.

The appearance of the first completely revised edition since the last war of this surgeon's vademecum is indeed a great event. The publishers are to be congratulated on the very high standard of the production, and Mr. Bailey's long hours of toil are amply rewarded.

Many chapters have been completely rewritten and numerous excellent illustrations (monochrome and colour) have been added. In this book we can obtain all the practical advice culled from writings and sayings of surgeons the world over, and the references are there at the end of the chapter for easy access.

In the appendix at the end one will find most of the useful tips and tricks which have been published since 1955 .

The volume sets out to be a reliable reference for the surgeon who is called upon to do emergency surgery. It achieves much more than this, for between its covers can be found the lore of the practical surgeon in any of the branches of surgery.

Certainly the young surgeon requires this textbook on his desk-not on his shelf. The older surgeon can spend many profitable hours browsing. through its pages.
G.F.C. 